\title{
ASSESSMENT OF PHYSICAL AND MECHANICAL PROPERTIES OF PAPUA NEW GUINEA TIMBER SPECIES
}

\author{
Benoit Belleville $^{1, \AA}$ Kilva Lancelot ${ }^{2}$, Elaine Galore $^{3,}$ Barbara Ozarska $^{1}$
}

\begin{abstract}
A comprehensive testing program has been developed to assess different physical and mechanical properties of 26 commercial and lesser-known Papua New Guinea species from secondary and plantation forests. The impact of log position in a tree on the mechanical properties has also been assessed to optimize the utilization of timbers along the value chain. The results showed that stiffness and bending strength tend to decrease or remain unchanged along the stem. Shear strength and Janka hardness displayed a similar trend to a lesser extent where the position in the tree had a limited impact on compression strength properties. Thus, segregating based on log position can be of interest where desired mechanical properties and costs associated with segregating justify optimum mechanical properties for the intended end use. The properties of selected species from plantations and regrowth forests were generally lower than those found in the literature for timbers from old-growth forests. The size of specimens tested, the amount and provenance of tested material, and some adaptive traits for tropical tree species are some factors potentially explaining observed differences. However, a comparison with recent studies tends to confirm the overall reduction of physical and mechanical properties when compared with old-growth forests timbers.
\end{abstract}

Keywords: Compression strength, flexural bending strength, hardness, plantations, regrowth forests, shear, stiffness.

\section{INTRODUCTION}

Papua New Guinea (PNG) is an Oceanian country located in the south-western Pacific Ocean region. The country has a total land area of 46,3 million hectares of which 33,6 million ha is estimated to contain forest cover (FAO 2015). Primary and regenerated forests represent $52,4 \%$ and $47,6 \%$ of the forest area, respectively, accounting for a total growing stock of 5195 million $\mathrm{m}^{3}$. According to the PNG Forest Authority, there are more than 2000 tree species in PNG, of which $20 \%$ are utilized in one way or another for commercial use (PNGFA 2007). Commercial forest plantations started in the 1960s with sporadic progress and currently covering 62277 ha. The country aims to develop 240000 ha of commercially viable and sustainable commercial forest plantations by the year 2030 to sustain 3,6 million $\mathrm{m}^{3}$ of industrial timber currently harvested from natural forest annually.

Although the forest cover in PNG is large and diverse, knowledge of the current timber resource is scarce. In the 1970s, an exhaustive review of the mechanical properties of PNG timbers from native forests has been conducted by Bolza and Kloot (1976). However, the most valuable commercial timbers have since been harvested in large areas of the primary rainforest as a result of forest policies focussing on export logging. Second-

\footnotetext{
${ }^{1}$ The University of Melbourne, Faculty of Science, School of Ecosystem and Forest Sciences, Richmond, Victoria, Australia.

${ }^{2}$ PNG Forest Research Institute, Lae, Morobe Province, Papua New Guinea.

${ }^{3}$ Timber and Forestry Training College, Lae, Morobe Province, Papua New Guinea.

•Corresponding author: benoit.belleville@unimelb.edu.au
}

Received: 12.12.2018 Accepted: 01.09.2019 
ary forests are recovering in terms of merchantable timber and carbon stocks but much of the potential timber resource is of lesser-known timber species.

Recent studies by Edwin and Ozarska (2015) and Kotlarewski et al. (2016) determined selected mechanical properties of some PNG timber species. However, there are still many research and structural challenges, constraints, and opportunities at different levels which need to be addressed to support the development of competitive value-added wood industries. The influence of environmental conditions and other growth factors on wood mechanical properties and the challenges associated with using this material has been the subject of numerous studies worldwide. Barrett and Kellogg (1991) noted a reduction in the mechanical properties of second growth Douglas-fir (Pseudotsuga menziesii) when compared to old-growth timber. Other studies also demonstrated that properties of wood produced from managed trees are different from wood produced in natural stands (Bendtsen 1978, Pearson and Gilmore 1980).

Currently, most local wood processing in PNG is focused on primary conversion of logs to low-grade building materials. Technical knowledge and capacity about efficient processing of different native timber species and produce a broader range of wood products are very low. The variability in properties of the wood material and of the finalized timber products indicates a potential for optimization too. Consequently, there is a need to provide research and technology development to develop and implement commercially sustainable log supply chains, knowledge, and capacity in wood science and processing technologies, as well as the processing structures which support successful domestic value-adding wood processing enterprises.

The aim of the project was to increase the contribution that utilization of forest resources makes to national and local economies, including landowners and processors, through the development of domestic value-added wood processing methods. The specific objectives were to 1) enhance the knowledge of wood properties of PNG timbers to facilitate greater value adding; 2) identify any relationship between log position in a tree and the mechanical properties of timber products to optimize the utilization of PNG timbers at various stages of the chain. Therefore, a testing program has been developed including the assessment of physical and mechanical properties of selected commercial and lesser-known species from secondary and plantation forests.

\section{MATERIALS AND METHODS}

\section{Harvesting}

The testing program has been divided into two groups of species to facilitate harvesting and specimens' preparation logistic. Each group included 13 species from plantations and regenerated forests (also known as regrowth forests) located in the Morobe and West New Britain provinces, PNG (Table 1). Nine species were harvested from plantations and 17 from regenerated forests ( 3 softwoods, 23 hardwoods). A total of 130 trees, i.e. 5 trees per species, have been selected and harvested in accordance with ASTM D5536 (2010). The trees have been selected based on the following selection criteria: 1) the tree had to be more than 15 years old after regrowth or planting; 2) all trees for a specific species had to be from the same forest area; 3 ) the selected trees had to be representative of the population, with good form and merchantable height. Following harvesting, the total merchantable height of each tree has been further cut into 3 to $4 \mathrm{~m}$-long logs, labeled as per height from ground (i.e. bottom, middle, and top section), and milled. The milled sawn boards were then kiln-dried to $12 \%$ moisture content. 
Table 1: PNG studied species information.

\begin{tabular}{|c|c|c|c|c|}
\hline Species & Trade Name & Origin & Age (years) & DBH $(\mathrm{cm})$ \\
\hline \multicolumn{5}{|l|}{ Plantations (9 species) } \\
\hline $\begin{array}{l}\text { Araucaria } \\
\text { cunninghamii }\end{array}$ & Pine, Hoop & Bulolo, Morobe & 28 & $39(4)$ \\
\hline Araucaria hunsteinii & Pine, Klinki & Bulolo, Morobe & 43 & $63(5)$ \\
\hline $\begin{array}{l}\text { Castanospermum } \\
\text { australe }\end{array}$ & Blackbean & $\begin{array}{l}\text { Kimbe, West } \\
\text { New Britain }\end{array}$ & 17 & $42(4)$ \\
\hline Eucalyptus deglupta & Kamarere & $\begin{array}{l}\text { Kimbe, West } \\
\text { New Britain }\end{array}$ & 29 & $60(14)$ \\
\hline Eucalyptus pellita & Pellita & Lae, Morobe & 18 & $36(5)$ \\
\hline Magnolia tsiampacca & Beech, Wau & Lae, Morobe & 17 & $34(4)$ \\
\hline Pinus caribaea & Pine, Caribbean & Lae, Morobe & 31 & $55(6)$ \\
\hline Pometia pinnata & Taun & Lae, Morobe & 18 & $48(6)$ \\
\hline Terminalia brassii & Terminalia, Brown & Lae, Morobe & 31 & $54(8)$ \\
\hline \multicolumn{5}{|c|}{ Secondary Forests / Regrowth (17 species) } \\
\hline Alstonia scholaris & Cheesewood, White & Lae, Morobe & 17 to 20 & $46(12)$ \\
\hline Anisoptera thurifera & Mersawa, PNG & Lae, Morobe & $+20^{2}$ & $46(8)$ \\
\hline $\begin{array}{l}\text { Anthocephalus } \\
\text { chinensis }\end{array}$ & Labula & Lae, Morobe & $+20^{*}$ & $51(8)$ \\
\hline Canarium oleosum & Canarium, Grey & Lae, Morobe & 17 to 20 & $40(2)$ \\
\hline $\begin{array}{l}\text { Elaeocarpus } \\
\text { sphaericus }\end{array}$ & Quandong, PNG & Lae, Morobe & 17 to 20 & $54(15)$ \\
\hline $\begin{array}{l}\text { Endospermum } \\
\text { medullosum }\end{array}$ & Basswood, PNG & Lae, Morobe & $+20^{2}$ & $47(14)$ \\
\hline Falcataria moluccana & Albizia, White & Lae, Morobe & $+20^{2}$ & $64(9)$ \\
\hline Homalium foetidum & Malas & Lae, Morobe & 17 to 20 & $52(16)$ \\
\hline Hopea iriana & Hopea, Heavy & Lae, Morobe & $+20^{*}$ & $44(4)$ \\
\hline Intsia bijuga & Kwila & Lae, Morobe & $+20^{2}$ & $40(4)$ \\
\hline Octomeles sumatrana & Erima & Lae, Morobe & $+20^{*}$ & $55(11)$ \\
\hline $\begin{array}{l}\text { Palaquium } \\
\text { warbargianum }\end{array}$ & Cedar, Pencil & Lae, Morobe & 17 to 20 & $43(2)$ \\
\hline Pangium edule & Pangium & Lae, Morobe & 17 to 20 & $38(3)$ \\
\hline Pterocarpus indicus & Rosewood, PNG & Lae, Morobe & $+20^{*}$ & $51(8)$ \\
\hline Syzygium spp. & Gum, Water & Lae, Morobe & 17 to 20 & $40(4)$ \\
\hline Vitex cofassus & Vitex, PNG & Lae, Morobe & $+20^{*}$ & $41(9)$ \\
\hline $\begin{array}{l}\text { Xanthophyllum } \\
\text { papuamum }\end{array}$ & Boxwood, PNG & Lae, Morobe & $+20^{*}$ & $42(9)$ \\
\hline
\end{tabular}

"No exact records of age are available in the harvested area. Species estimated age is +20 years old. Standard deviation in parentheses.

\section{Machining specimens and mechanical testing}

Specimens have then been machined in accordance with ASTM D143 (2009) and conditioned to $23^{\circ} \mathrm{C}$ and $65 \%$ relative humidity until constant mass prior to testing. Whenever possible and applicable, a $50 \times 50$ $\mathrm{mm}$ specimen cross-section size has been selected. The selected mechanical properties for the present study were stiffness (MOE), flexural bending strength (MOR), compression strength parallel and perpendicular to the grain, shear strength parallel to the grain, and hardness (Janka). All the tests have been conducted using a universal testing machine (Instron model 5569, MA, USA). Prior to testing, each specimen has been measured to determine the volume and weighted to determine mass at the time of testing.

After every test, a section has been taken from the specimen near the point of failure, weighted, and placed at $103^{\circ} \mathrm{C}$ for 24 hours to determine the oven-dry mass and moisture content.

Static bending test (MOE and MOR): 20 specimens of dimensions 50 x 50 x $760 \mathrm{~mm}$ have been prepared per species and tested using a 3-point bending rig. The specimens have been tested using a loading speed of $2,5 \mathrm{~mm} / \mathrm{min}$ until failure.

Compression strength parallel to the grain: 20 specimens of dimensions $25 \times 25 \times 100 \mathrm{~mm}$ have been prepared and tested per species. The speed of testing was $0,3 \mathrm{~mm} / \mathrm{min}$.

Compression strength perpendicular to the grain: 20 specimens of dimensions $50 \times 50 \times 200 \mathrm{~mm}$ have been 
prepared per species. The speed of testing was $0,6 \mathrm{~mm} / \mathrm{min}$.

Shear strength parallel to the grain: 20 specimens of dimensions $50 \times 50 \times 63 \mathrm{~mm}$ have been prepared and tested per species. The speed of testing was $0,6 \mathrm{~mm} / \mathrm{min}$. The portion of the piece that was sheared off has been used as a moisture content specimen.

Janka hardness: 20 specimens of dimensions 50 x 50 x $150 \mathrm{~mm}$ have been prepared and tested per species. The number of test penetrations included two (2) on a tangential surface, two (2) on a radial surface, and one (1) on each end. Only indentations on tangential and radial surfaces have been used to calculate the Janka hardness value. The speed of testing was $6 \mathrm{~mm} / \mathrm{min}$.

\section{Statistical analysis}

The data were analyzed in Minitab statistical software (Minitab 18, Version 18.1) to evaluate the effect within species of the position in a tree on the mechanical properties. A fit mixed effects model and a Fisher pairwise comparisons analysis $(\alpha=0,05)$ were used. In the model, the tree identification number (tree \#1 to \#5) was used as a random factor where the species and position in the tree were the fixed factors.

\section{RESULTS AND DISCUSSION}

A total of 2,641 specimens from 26 species and 130 trees have been tested i.e. shear: 528 specimens; compression parallel: 526 specimens; compression perpendicular: 532 specimens; static bending: 525 specimens; hardness: 530 specimens. The total merchantable height of Magnolia tsiampacca trees was usually too short to obtain top sections. Therefore, only bottom and middle sections could generally be obtained for this species which has been excluded from the model.

Hopea iriana provided significantly higher mechanical testing results than any other species across all selected mechanical properties (Table 2). A group composed of Eucalyptus pellita, Homalium foetidum, and Intsia bijuga usually performed significantly better than the other tested species. Xanthophyllum papuanum, Anisoptera thurifera, and Castanospermum australe also typically performed above average. At the other end, a group formed of Octomeles sumatrana, Falcataria moluccana, Endospermum medullosum, Alstonia scholaris, Palaquium warbargianum, Elaeocarpus sphaericus, and Magnolia tsiampacca generally offered mechanical properties below the average.

Table 2: Summary of mechanical properties per species.

\begin{tabular}{|c|c|c|c|c|c|c|}
\hline & MOE & MOR & $\begin{array}{l}\text { Cor } \\
\text { Parallel to }\end{array}$ & $\begin{array}{l}\text { ession } \\
\text { Perpendicular }\end{array}$ & $\begin{array}{l}\text { Shear } \\
\text { Parallel to grain }\end{array}$ & $\begin{array}{c}\text { Hardness } \\
\text { Janka }\end{array}$ \\
\hline Species & GPa & MPa & $\begin{array}{l}\text { grain } \\
\text { MPa }\end{array}$ & $\begin{array}{l}\text { tograin } \\
\text { NPa }\end{array}$ & MPa & $N$ \\
\hline $\begin{array}{l}\text { Alstonia a cholaris } \\
\text { Fisher LSD }\end{array}$ & $\begin{array}{c}5,1 \\
Q\end{array}$ & $\frac{33.4}{\mathrm{R}}$ & $\begin{array}{l}18.7 \\
\mathrm{M}\end{array}$ & $\begin{array}{l}3.5 \\
\mathrm{MN}\end{array}$ & 4.6 & $\begin{array}{l}896 \\
\text { IJ }\end{array}$ \\
\hline $\begin{array}{l}\text { Arisopptera thurifera } \\
\text { Fisher LSD }\end{array}$ & $\begin{array}{l}14,6 \\
\mathrm{CD}\end{array}$ & $\begin{array}{c}86,0 \\
\mathrm{EF}\end{array}$ & $\begin{array}{l}51,2 \\
D E\end{array}$ & $\frac{9,3}{\mathrm{~F}}$ & $\begin{array}{c}10,4 \\
D\end{array}$ & $\underset{E}{3797}$ \\
\hline 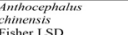 & $\begin{array}{l}7,7 \\
\text { LMN }\end{array}$ & $\begin{array}{l}56,5 \\
\text { LMN }\end{array}$ & $\begin{array}{l}31,4 \\
\mathrm{IJ}\end{array}$ & $\begin{array}{l}6,8 \\
\mathrm{HI}\end{array}$ & $\begin{array}{l}7,5 \\
\mathrm{HI}\end{array}$ & $\begin{array}{c}2179 \\
\mathrm{G}\end{array}$ \\
\hline 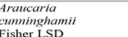 & $\begin{array}{l}8,1 \\
\mathrm{LM}\end{array}$ & $\begin{array}{l}60,1 \\
\text { KLM }\end{array}$ & $\stackrel{24,4}{L}$ & $\begin{array}{l}7,5 \\
\mathrm{GH}\end{array}$ & $\begin{array}{l}7,7 \\
\mathrm{GH}\end{array}$ & $\underset{\mathrm{G}}{2229}$ \\
\hline $\begin{array}{l}\text { Fisher LSD } \\
\text { Arancaria humsteinii } \\
\text { Fisher LSD }\end{array}$ & $\begin{array}{l}10.6 \\
\mathrm{HI}\end{array}$ & $\begin{array}{c}68.9 \\
\mathrm{IJ}\end{array}$ & $\stackrel{31.0}{1 j}$ & $\begin{array}{l}7.5 \\
\mathrm{GH}\end{array}$ & $\begin{array}{l}8.6 \\
\text { EFG }\end{array}$ & $\underset{G}{2121}$ \\
\hline $\begin{array}{l}\text { Fisher LSD } \\
\text { Camariumm oleosum } \\
\text { Fisher LSD }\end{array}$ & $\begin{array}{l}9,3 \\
\mathrm{JK}\end{array}$ & $\begin{array}{l}62,3 \\
1 \mathrm{KL}\end{array}$ & $\begin{array}{c}33,5 \\
\mathrm{HI}\end{array}$ & $\begin{array}{l}72 \\
\text { GHI }\end{array}$ & $\begin{array}{l}8,5 \\
\mathrm{E}, 5 \mathrm{G}\end{array}$ & $\begin{array}{c}2177 \\
\mathrm{G}\end{array}$ \\
\hline $\begin{array}{l}\text { Castianospermut } \\
\text { acustrale } \\
\text { Fisher LSD }\end{array}$ & $\begin{array}{l}11,5 \\
\text { FG }\end{array}$ & $\begin{array}{l}85,2 \\
\mathrm{EF}\end{array}$ & $\begin{array}{l}48,2 \\
\mathrm{EF}\end{array}$ & $\begin{array}{l}12,7 \\
\text { D }\end{array}$ & $\begin{array}{c}10,9 \\
D\end{array}$ & $\begin{array}{l}4490 \\
D\end{array}$ \\
\hline $\begin{array}{l}\text { Elacocarpus } \\
\text { phpaepicus } \\
\text { Fisher LSD }\end{array}$ & $\begin{array}{l}7,7 \\
\text { LMN }\end{array}$ & $\begin{array}{l}50,5 \\
\text { NOP }\end{array}$ & $\begin{array}{l}28,6 \\
J K\end{array}$ & $\begin{array}{l}4,9 \\
K L\end{array}$ & $\underset{1}{6,6}$ & $\begin{array}{c}1554 \\
\mathrm{H}\end{array}$ \\
\hline $\begin{array}{l}\text { Endospermum } \\
\text { medullosum } \\
\text { Fisher LSD }\end{array}$ & $\begin{array}{l}7,8 \\
\text { LMN }\end{array}$ & $\begin{array}{l}44,9 \\
P Q\end{array}$ & $\stackrel{29,5}{j}$ & $\begin{array}{l}4,2 \\
L M\end{array}$ & $\begin{array}{l}5,4 \\
\text { JK }\end{array}$ & $\begin{array}{c}1311 \\
\mathrm{HI}\end{array}$ \\
\hline $\begin{array}{l}\text { Escaly prots deghipta } \\
\text { Fisher LSD }\end{array}$ & $\begin{array}{l}8,6 \\
\text { KL }\end{array}$ & $\begin{array}{l}62,1 \\
\mathrm{JKL}\end{array}$ & $\begin{array}{c}31,33 \\
\mathrm{I}, \mathrm{j}\end{array}$ & $\begin{array}{c}9,5 \\
\mathrm{~F}\end{array}$ & $\begin{array}{l}8,6 \\
E F G\end{array}$ & $\begin{array}{c}3132 \\
F\end{array}$ \\
\hline $\begin{array}{l}\text { Eucalypurts pellita } \\
\text { Fisher LSD }\end{array}$ & $\begin{array}{l}\frac{1}{15,6} \\
\mathrm{~B}\end{array}$ & $\begin{array}{l}120,6 \\
C \mathrm{C}\end{array}$ & $\begin{array}{c}52,7 \\
\mathrm{D}\end{array}$ & 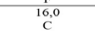 & $\begin{array}{c}12,3 \\
c\end{array}$ & $\begin{array}{l}6215 \\
C\end{array}$ \\
\hline $\begin{array}{l}\text { Falcataria moluccana } \\
\text { Fisher LSD }\end{array}$ & $\begin{array}{l}6,7 \\
O P\end{array}$ & $\begin{array}{l}42,7 \\
Q\end{array}$ & $\begin{array}{l}25,5 \\
\mathrm{KL}\end{array}$ & 4,1 & 5,6 & $\begin{array}{l}1192 \\
H 11\end{array}$ \\
\hline $\begin{array}{l}\text { Fomalinum foetidum } \\
\text { Homation }\end{array}$ & $\begin{array}{l}15,4 \\
B C\end{array}$ & $\frac{Q}{128,4}$ & $\begin{array}{l}\mathrm{RL} \\
58,6\end{array}$ & $\begin{array}{l}\mathrm{LM} \\
15,5 \\
\mathrm{C}\end{array}$ & B & $\begin{array}{l}\mathrm{HIJ} \\
6893\end{array}$ \\
\hline 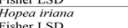 & 20,0 & 136,9 & 69,0 & 20,6 & $\begin{array}{c}13,5 \\
16,5\end{array}$ & $\frac{1553}{8753}-10$ \\
\hline $\begin{array}{l}\text { Fisher LSD } \\
\text { Intisia bijuga } \\
\text { Fisho isD }\end{array}$ & $\begin{array}{l}\text { A } \\
14,2\end{array}$ & $\frac{A}{116,6}$ & $\begin{array}{l}\mathrm{A} \\
64,1 \\
\mathrm{~B}\end{array}$ & $\begin{array}{l}\mathrm{A} \\
17.3 \\
\mathrm{~B}\end{array}$ & $\begin{array}{l}\mathrm{A} \\
13,5 \\
\mathrm{~B}\end{array}$ & $\begin{array}{l}\mathrm{A} \\
6361\end{array}$ \\
\hline $\begin{array}{l}\text { Fisher LSD } \\
\text { Magnolia ssiampacca }\end{array}$ & $\frac{D}{6,1}$ & $\frac{c}{48,5}$ & $\begin{array}{c}\mathrm{B} \\
25,4\end{array}$ & $\frac{B}{5.6}$ & $\frac{B}{5.5}$ & $\frac{B C}{1273}$ \\
\hline $\begin{array}{l}\text { Fisher LSD } \\
\text { Ociomeles sumatrana }\end{array}$ & $\begin{array}{c}p \\
4,8\end{array}$ & $\begin{array}{l}\mathrm{OPQ} \\
31,5\end{array}$ & $\begin{array}{l}\mathrm{KL} \\
18,5\end{array}$ & $\begin{array}{l}3 \mathrm{KK} \\
2,7\end{array}$ & $\begin{array}{l}J \mathrm{JK} \\
3,6 \\
j\end{array}$ & $\begin{array}{l}\text { HIJ } \\
735\end{array}$ \\
\hline $\begin{array}{l}\text { Fisher LSD } \\
\text { Paldugumitm }\end{array}$ & Q & $\mathrm{s}_{\mathrm{s}}$ & M & & & \\
\hline $\begin{array}{l}\text { Palaquanmm } \\
\text { warbargianumn } \\
\text { Fisher ISD }\end{array}$ & $\begin{array}{l}7,5 \\
\text { MNO }\end{array}$ & $\begin{array}{l}50,8 \\
\text { NOP }\end{array}$ & $\begin{array}{l}25,4 \\
\mathrm{KL}\end{array}$ & $\begin{array}{l}4,5 \\
\text { KLM }\end{array}$ & $\begin{array}{l}5,5 \\
\mathrm{JK}\end{array}$ & ${ }_{\mathrm{HI}}^{1374}$ \\
\hline $\begin{array}{l}\text { Fisher LSD } \\
\text { Pangium cdule } \\
\text { Fisher LSD }\end{array}$ & $\underset{\mathrm{F}}{12,1}$ & $\begin{array}{l}70,1 \\
\mathrm{HL}\end{array}$ & $\begin{array}{l}36,7 \\
\mathrm{GH}\end{array}$ & $\begin{array}{l}7,5 \\
\mathrm{GH}\end{array}$ & $\begin{array}{l}8,0 \\
\text { FGH }\end{array}$ & $\begin{array}{c}3091 \\
F\end{array}$ \\
\hline 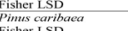 & 8,0 & 67,2 & $\begin{array}{l}291 \\
291 \\
31\end{array}$ & $\begin{array}{l}8,3 \\
6\end{array}$ & 垔, & $\frac{r}{2311}$ \\
\hline $\begin{array}{l}\text { Fisher LSD } \\
\text { Pomelip pimata }\end{array}$ & $\frac{\mathrm{LM}}{11,1}$ & $\begin{array}{l}10 \mathrm{~K} \\
91,1\end{array}$ & $\frac{J K}{36,9}$ & $\frac{6}{12,7}$ & $\frac{E}{11,2}$ & $\frac{G}{4904}$ \\
\hline 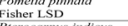 & $\mathrm{GH}$ & $\mathrm{DE}$ & $\mathrm{GH}$ & D & D & D \\
\hline $\begin{array}{l}\text { Peterocarplus indicicus } \\
\text { Fisher LSD }\end{array}$ & $\begin{array}{c}10,0 \\
\text { IJ }\end{array}$ & $\begin{array}{l}76,1 \\
\mathrm{GH}\end{array}$ & $\begin{array}{c}45,7 \\
F\end{array}$ & $\begin{array}{c}11,4 \\
\mathrm{E}\end{array}$ & $\begin{array}{c}9,3 \\
\mathrm{E}\end{array}$ & $\begin{array}{l}3239 \\
\mathrm{~F}\end{array}$ \\
\hline 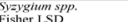 & 9,6 & 68.1 & $\begin{array}{c}38.3 \\
\mathrm{G}\end{array}$ & 8.2 & $\begin{array}{l}8.7 \\
\mathrm{FE}\end{array}$ & 2410 \\
\hline Terminaliab brassit & 6,9 & 54,4 & 29.9 & 6,0 & 8,5 & 2518 \\
\hline $\begin{array}{l}\text { Fisher LSD } \\
\text { Viter coffesus }\end{array}$ & NOP & $\begin{array}{l}\text { MNO } \\
80.8\end{array}$ & $\frac{15}{459}$ & $\begin{array}{l}1 \mathrm{~J} \\
100\end{array}$ & EFG & \\
\hline Fisher LSD & $\begin{array}{l}\text { FGH } \\
\text { FGH }\end{array}$ & $\begin{array}{l}\text { FG } \\
\text { FG }\end{array}$ & F & $\mathrm{F}$ & E & $\begin{array}{l}3.72 \\
\mathrm{EF}\end{array}$ \\
\hline $\begin{array}{l}\text { Xanthophy/lum } \\
\text { papuamum }\end{array}$ & $\begin{array}{c}13,1 \\
\text { E }\end{array}$ & $\begin{array}{c}94,2 \\
\mathrm{D}\end{array}$ & 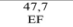 & 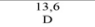 & 10,6 & $\begin{array}{c}4899 \\
\mathrm{D}\end{array}$ \\
\hline
\end{tabular}

MOE: Modulus of elasticity or stiffness; MOR: Modulus of rupture or bending strength. 


\section{Effect of position in the tree on selected physical and mechanical properties}

The physical and static bending properties per species based on the position in the tree are presented in Table 3 . The statistical analysis demonstrated a very significant interaction $(P$-value $<0,001)$ between species and position in the tree on the bending properties. Stiffness and bending strength tend to decrease or remain unchanged along the stem across all studied species. The average stiffness and bending strength values obtained from bottom and middle sections were significantly greater than those from top sections for 6 (Araucaria cunninghamii, Canarium oleosum, Hopea iriana, Intsia bijuga, Pangium edule, Xanthophyllum papuanum) and 5 (Canarium oleosum, Homalium foetidum, Intsia bijuga, Pangium edule, Xanthophyllum papuanum) species, respectively. Such a trend was not noticeable in species typically performing below the average of all tested species. This observation is in accordance with other studies reported in the literature (Harvald 1988, Shivnaraine 1989, Hojbo 1991, Kliger et al. 1995, Machado and Cruz 2005). In one case, i.e. Eucalyptus pellita, top sections provided significantly higher bending properties than bottom sections. Such a result might be explained by the fact that specimens were taken from logs without any consideration to the radial position within the tree. Consequently, some specimens obtained from bottom sections might have included a higher proportion of juvenile wood, usually not as strong as mature wood, if taken from the outer radial section of the tree and vice-versa. Kliger et al. (1995) also reported that strength and stiffness in butt logs usually tend to increase further away from the pith in the radial direction.

Table 3: Physical and bending properties per species and position in the tree (i.e. bottom, middle, or top section based on height from ground).

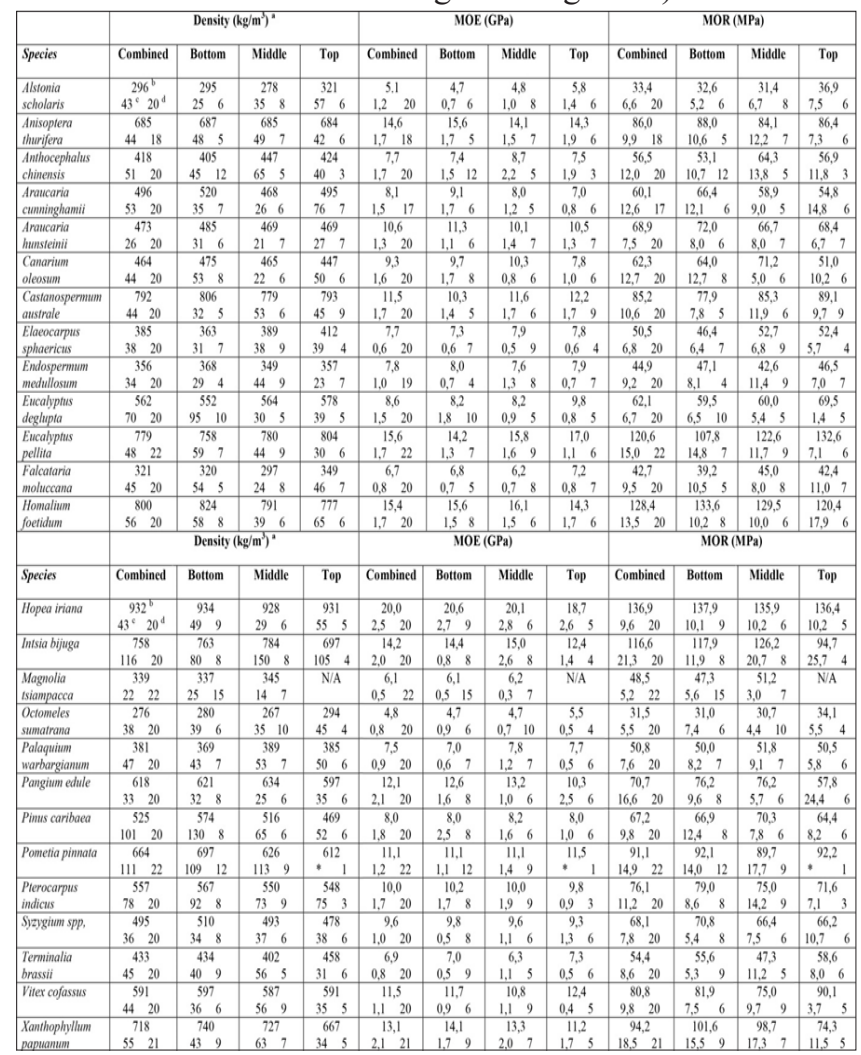

${ }^{\mathrm{a}}$ Air dry density at $12 \%$ moisture content; ${ }^{\mathrm{b}} \mathrm{Mean}$; ${ }^{\mathrm{c}} \mathrm{Standard}$ deviation; ${ }^{\mathrm{d}}$ Number of specimens.

The position in the tree had a more limited impact on the compression strength properties. The species and position in tree interaction significantly influenced the compression perpendicular to grain in the case of two species, i.e. Eucalyptus deglupta and Pterocarpus indicus (Table 4). In both cases, top sections provided significantly higher average values than bottom sections $(P$-value $<0,020)$. In the case of compression parallel to the grain, the crushing strength was not significantly influenced by the position in the tree for any of the tested species. The Fisher pairwise comparisons analysis identified two species, i.e. Castanospermum australe and Anisoptera thurifera, where the bottom sections provided significantly higher results than the top sections. 
Table 4: Compression parallel and perpendicular to the grain per species based on position in the tree (based on height from ground).

\begin{tabular}{|c|c|c|c|c|c|c|c|c|}
\hline & \multicolumn{4}{|c|}{ Compression Parallel to Grain (MPa) } & \multicolumn{4}{|c|}{ Compression Perpendicular to Grain (MPa) } \\
\hline Species & Combined & Bottom & Middle & Top & Combined & Bottom & Middle & Top \\
\hline $\begin{array}{l}\text { Alstonia } \\
\text { scholaris }\end{array}$ & $\begin{array}{c}18,7^{\mathrm{a}} \\
2,4^{\mathrm{b}} 20^{\mathrm{c}}\end{array}$ & $\begin{array}{ll} & 18,5 \\
2,5 & 6\end{array}$ & \begin{tabular}{ll}
\multicolumn{2}{c}{18,3} \\
1,2 & 6
\end{tabular} & $\begin{array}{ll}19,1 & \\
3,2 & 8\end{array}$ & $0,7^{3,5} 20$ & $\begin{array}{ll}3,3 \\
0,7 & 7\end{array}$ & $\begin{array}{ll}3,3 \\
0,4 \quad 7\end{array}$ & $\begin{array}{ll} & 4,0 \\
0,9 & 6\end{array}$ \\
\hline $\begin{array}{l}\text { Anisoptera } \\
\text { thurifera }\end{array}$ & $\begin{array}{c}51,2 \\
3,9 \quad 20\end{array}$ & $\begin{array}{l}54,8 \\
3,0 \quad 8\end{array}$ & $\begin{array}{c}49,5 \\
2,2 \quad 7\end{array}$ & $\begin{array}{c}47,9 \\
2,5\end{array}$ & $2,0^{9,3} 20$ & $\begin{array}{c}10,1 \\
2,0 \quad 7\end{array}$ & \begin{tabular}{ll}
\multicolumn{1}{c}{9,3} \\
$2,6 \quad 7$
\end{tabular} & $\begin{array}{cc}8,4 \\
0,5\end{array}$ \\
\hline $\begin{array}{l}\text { Anthocephatus } \\
\text { chinensis }\end{array}$ & $\begin{array}{c}31,4 \\
6,7^{2} 20\end{array}$ & $\begin{array}{l}28,0 \\
6,6 \quad 5\end{array}$ & $\begin{array}{c}32,8 \\
6,4 \quad 7\end{array}$ & $\begin{array}{l}32,3 \\
7,1\end{array}$ & $\begin{array}{c}6,8 \\
1,9\end{array}$ & $\begin{array}{c}6,4 \\
2,4\end{array}$ & $\begin{array}{r}7,1 \\
1,3\end{array}$ & $\begin{array}{c}7,2 \\
2,6\end{array}$ \\
\hline $\begin{array}{l}\text { Araucaria } \\
\text { cumninghamii }\end{array}$ & $3,8 \quad 24,4$ & $\begin{array}{l}25,5 \\
5,0 \quad 5\end{array}$ & $\begin{array}{l}22,6 \\
3,4 \quad 7\end{array}$ & $\begin{array}{l}25,2 \\
3,2 \quad 8\end{array}$ & $1,5^{7,5} 20$ & $\begin{array}{l}7,9 \\
1,5 \quad 7\end{array}$ & $\begin{array}{l}6,5 \\
1,2\end{array}$ & $\begin{array}{c}8,0 \\
1,5 \quad 7\end{array}$ \\
\hline $\begin{array}{l}\text { Araucaria } \\
\text { hrunsteinit }\end{array}$ & $\begin{array}{l}31,0 \\
2,2 \quad 20\end{array}$ & $\begin{array}{c}31,4 \\
1,6 \quad 7\end{array}$ & $\begin{array}{c}31,3 \\
3,0 \quad 5\end{array}$ & $2,30,4$ & $0,6^{7,5} 20$ & $\begin{array}{ll} & 7,5 \\
1,0 & 6\end{array}$ & $\begin{array}{l}7,7 \\
0,4 \quad 8\end{array}$ & $\begin{array}{ll} & 7,2 \\
0,4 & 6\end{array}$ \\
\hline $\begin{array}{l}\text { Canarium } \\
\text { oleosum }\end{array}$ & $\begin{array}{c}33,5 \\
5,1 \quad 20\end{array}$ & $\begin{array}{c}32,3 \\
3,4 \quad 7\end{array}$ & $\begin{array}{c}39,0 \\
2,1\end{array}$ & $\begin{array}{l}30,0 \\
4,4 \quad 7\end{array}$ & $1,4,20$ & $\begin{array}{l}7,5 \\
1,5\end{array}$ & $\begin{array}{c}7,7 \\
1,6\end{array}$ & $\begin{array}{cc}6,1 \\
0,6\end{array}$ \\
\hline $\begin{array}{l}\text { Castanospermu } \\
\text { m australe }\end{array}$ & $\begin{array}{c}48,2 \\
12,4 \quad 20\end{array}$ & $\begin{array}{c}53,6 \\
17,3 \quad 8\end{array}$ & $\begin{array}{c}44,7 \\
8,3 \quad 6\end{array}$ & $\begin{array}{l}44,7 \\
4,9 \quad 6\end{array}$ & $1,2 \quad 20$ & $\begin{array}{c}13,3 \\
1,5 \quad 6\end{array}$ & $\begin{array}{c}12,5 \\
1,1 \quad 7\end{array}$ & $\begin{array}{c}12,4 \\
0,7\end{array}$ \\
\hline $\begin{array}{l}\text { Elaeocarpus } \\
\text { sphaericus }\end{array}$ & $\begin{array}{c}28,6 \\
2,6 \quad 20\end{array}$ & $\begin{array}{l}26,0 \\
0,7 \quad 5\end{array}$ & $\begin{array}{c}29,4 \\
2,2\end{array}$ & $\begin{array}{ll}29,6 & \\
3,1 & 6\end{array}$ & $1,2,20$ & $\begin{array}{cc} & 4,1 \\
0,7 & 7\end{array}$ & $\begin{array}{ll}5,1 & \\
1,5 & 7\end{array}$ & $1,0^{5,6} 6$ \\
\hline $\begin{array}{l}\text { Endospermum } \\
\text { medullosum }\end{array}$ & $\begin{array}{c}29,5 \\
5,0 \quad 20\end{array}$ & $\begin{array}{l}28,6 \\
6,2 \quad 7\end{array}$ & $\begin{array}{c}28,9 \\
5,0 \quad 8\end{array}$ & $\begin{array}{ll}3,1,6 \\
3,1 & 5\end{array}$ & $0,8^{4,2} 20$ & $\begin{array}{ll} & 4,5 \\
0,6 & 8\end{array}$ & $\begin{array}{c}3,5 \\
1,2\end{array}$ & $\begin{array}{c}4,1 \\
0,7\end{array}$ \\
\hline $\begin{array}{l}\text { Eucalyptus } \\
\text { deglupta }\end{array}$ & $\begin{array}{l}31,3 \\
9,5 \\
20\end{array}$ & $\begin{array}{l}31,2 \\
3,6 \quad 7\end{array}$ & $\begin{array}{l}30,5 \\
8,0 \quad 8\end{array}$ & $\begin{array}{c}32,8 \\
17,2\end{array}$ & $2,5^{9,5} 21$ & $2,0^{8,4} 11$ & $\begin{array}{l}10,3 \\
1,1\end{array}$ & $\begin{array}{l}11,1 \\
3,8 \quad 5\end{array}$ \\
\hline $\begin{array}{l}\text { Eucalyptus } \\
\text { pellita }\end{array}$ & 52,7 & $\begin{array}{l}51,6 \\
7,4\end{array}$ & $\begin{array}{ll}52,4 \\
105 & 6\end{array}$ & $\begin{array}{ll}54,6 \\
11,4\end{array}$ & $\begin{array}{ll}16,0 \\
30\end{array}$ & $\begin{array}{l}16,2 \\
3,7\end{array}$ & $\begin{array}{l}15,8 \\
2,5\end{array}$ & $\begin{array}{c}15,8 \\
2.6\end{array}$ \\
\hline $\begin{array}{l}\text { Falcataria } \\
\text { moluccana }\end{array}$ & $3,5 \quad 25$ & $3,84,4$ & $2,7 \quad 12$ & $\begin{array}{l}28,7 \\
4,1\end{array}$ & $1,5,1$ & $\begin{array}{ll}2,7 & \\
0,8 & 5\end{array}$ & $1,2^{4,5} \quad 12$ & $\begin{array}{ll}4,6 \\
2,1 & 4\end{array}$ \\
\hline \multirow[t]{2}{*}{$\begin{array}{l}\text { Homalinum } \\
\text { foetidum }\end{array}$} & $\begin{aligned} 58,6 \\
8,8\end{aligned}$ & $\begin{array}{c}59,9 \\
7,3 \quad 7\end{array}$ & $\begin{array}{c}58,7 \\
14,1 \quad 6\end{array}$ & $\begin{array}{c}57,3 \\
5,0 \quad 7\end{array}$ & $2,5 \quad 20$ & $2,5,7$ & $\begin{array}{l}15,4 \\
2,4\end{array}$ & $\begin{array}{l}14,5 \\
2,7\end{array}$ \\
\hline & \multicolumn{4}{|c|}{ Compression Parallel to Grain (MPa) } & \multicolumn{4}{|c|}{ Compression Perpendicular to Grain (MPa) } \\
\hline Species & Combined & Bottom & Middle & Top & Combined & Bottom & Middle & Top \\
\hline Hopea iriana & $\begin{array}{c}69,0^{\mathrm{a}} \\
6,8^{\mathrm{b}} 20^{\mathrm{c}}\end{array}$ & $\begin{array}{c}69,5 \\
10,0\end{array}$ & $\begin{array}{ll}68,3 \\
5,8 & 7\end{array}$ & ${ }_{5,3}^{69,4} 7$ & $\begin{array}{l}20,6 \\
0,5\end{array}$ & $\begin{array}{l}20,7 \\
0,5 \quad 8\end{array}$ & $\begin{array}{l}20,6 \\
0,5\end{array}$ & $\mathrm{O}_{0,5}^{20,4} 5$ \\
\hline Imsia bijuga & $\begin{array}{c}64,1 \\
5,3 \quad 20\end{array}$ & $\begin{array}{c}66,6 \\
7,7 \quad 7\end{array}$ & $\begin{array}{c}62,5 \\
3,2 \\
3,2\end{array}$ & $\begin{array}{l}64,2 \\
4,4 \quad 2\end{array}$ & $\begin{array}{l}17,3 \\
3,3 \quad 17\end{array}$ & $\begin{array}{c}17,0 \\
3,6 \quad 10\end{array}$ & $\begin{array}{c}19,2 \\
1,8 \quad 4\end{array}$ & $\begin{array}{c}15,6 \\
3,4 \quad 3\end{array}$ \\
\hline $\begin{array}{l}\text { Magnolia } \\
\text { tsiampacca }\end{array}$ & $2,7{ }_{20}^{25,4}$ & $2,24,6$ & $\begin{array}{l}25,9 \\
3,1\end{array}$ & ${ }_{*}^{28,8}{ }_{1}$ & $0,9^{5,6} 22$ & $1,00^{5,7} 10$ & $0,5,39$ & $\begin{array}{ll}6,0 \\
0.5\end{array}$ \\
\hline $\begin{array}{l}\text { Octomeles } \\
\text { sumatrana }\end{array}$ & $3,2 \quad 19$ & $\begin{array}{c}17,1 \\
3,1 \quad 7\end{array}$ & $\begin{array}{c}19,1 \\
2,6\end{array}$ & $\begin{array}{l}19,5 \\
3,6 \quad 7\end{array}$ & $0,9^{2,7} 20$ & $0,7 \quad 6$ & $0,8^{2,6} \quad 10$ & $\begin{array}{ll}3,2 \\
1,2\end{array}$ \\
\hline $\begin{array}{l}\text { Palaquium } \\
\text { warbargiamum }\end{array}$ & $2,7 \quad 25$ & $\begin{array}{l}25,1 \\
1,7\end{array}$ & $\begin{array}{l}25,6 \\
2,8\end{array}$ & $\begin{array}{l}25,4 \\
3,9\end{array}$ & $1,2^{4,5} 20$ & $\begin{array}{l}4,4 \\
1,68\end{array}$ & $\begin{array}{ll}4,6 \\
1,0 & 6\end{array}$ & $\begin{array}{l}4,5 \\
0,8\end{array}$ \\
\hline Pangium edule & $\begin{array}{c}36,7 \\
7,6 \quad 20\end{array}$ & $\begin{array}{ll}37,5 \\
9,0 & 6\end{array}$ & $\begin{array}{c}39,2 \\
6,4 \quad 7\end{array}$ & $\begin{array}{l}33,5 \\
7,3 \quad 7\end{array}$ & $1,2^{7,5} 20$ & $\begin{array}{c}8,0 \\
1,4 \quad 8\end{array}$ & $\begin{array}{ll} & 7,6 \\
0,4 \quad 5\end{array}$ & $\begin{array}{cc}6,6,8 \\
0,9\end{array}$ \\
\hline Pimus caribaea & $\begin{array}{c}29,1 \\
6,0 \quad 20\end{array}$ & $\begin{array}{l}26,5 \\
3,8 \quad 7\end{array}$ & $\begin{array}{c}31,7 \\
6,3 \quad 9\end{array}$ & $\begin{array}{c}27,8 \\
7,5 \quad 4\end{array}$ & $1,8,3$ & $\begin{array}{ll} & 8,0 \\
0,5 & 7\end{array}$ & $\begin{array}{c}8,4 \\
1,7 \quad 2\end{array}$ & \begin{tabular}{c}
\multicolumn{2}{c}{8,5} \\
$1,9 \quad 11$
\end{tabular} \\
\hline Pometia pinnata & $\begin{array}{c}36,9 \\
4,9 \quad 21\end{array}$ & $\begin{array}{c}37,3 \\
6,0 \quad 8\end{array}$ & $\begin{array}{cc}35,6 \\
5,5 & 5\end{array}$ & $3,8^{37,3} 8$ & $\begin{array}{l}12,7 \\
3,3^{2}\end{array}$ & $\begin{array}{c}12,2 \\
2,7 \quad 12\end{array}$ & $\begin{array}{l}14,1 \\
3,9 \quad 8\end{array}$ & $3,2^{10,5} 2$ \\
\hline $\begin{array}{l}\text { Pterocarpus } \\
\text { irdicus }\end{array}$ & $7_{7,2}^{45,7} 21$ & $\begin{array}{l}45,8 \\
6,5 \quad 7\end{array}$ & $\begin{array}{l}45,2 \\
6,9\end{array}$ & $\begin{array}{l}46,2 \\
9,2\end{array}$ & $2,2^{11,4} 21$ & $\begin{array}{l}10,7 \\
2,5\end{array}$ & $\begin{array}{l}11,4 \\
1,6\end{array}$ & $\begin{array}{l}13,2 \\
2,4\end{array}$ \\
\hline Syzygium spp & $\begin{array}{c}38,3 \\
5,6 \quad 20\end{array}$ & $\begin{array}{r}34,6 \\
4,9 \quad 5\end{array}$ & $\begin{array}{c}40,3 \\
6,7 \quad 8\end{array}$ & $\begin{array}{l}38,7 \\
3,5 \quad 7\end{array}$ & $\begin{array}{l}8,2 \\
1,8 \quad 20\end{array}$ & $\begin{array}{c}7,8 \\
2,2 \quad 8\end{array}$ & $\begin{array}{c}8,9 \\
1,3\end{array}$ & $\begin{array}{cc}8,1 \\
1,6\end{array}$ \\
\hline Termi & 29,9 & 29,0 & 29,9 & 32,0 & 6,0 & 5,5 & 3,7 & 6,9 \\
\hline brassii & $4,4 \quad 20$ & $5,2 \quad 10$ & $3,5 \quad 6$ & $3,5 \quad 4$ & $1,3 \quad 20$ & $1,2 \quad 9$ & $0,1 \quad 2$ & $0,6 \quad 9$ \\
\hline Vitex cofassus & $\begin{array}{c}45,9 \\
5,4 \quad 20\end{array}$ & $\begin{array}{l}46,5 \\
6,1 \quad 11\end{array}$ & $\begin{array}{c}42,0 \\
4,6 \quad 4\end{array}$ & $\begin{array}{l}47,5 \\
3,1 \quad 5\end{array}$ & $\begin{array}{c}10,0 \\
1,8 \quad 20\end{array}$ & $\begin{array}{c}9,8 \\
2,0\end{array}$ & $\begin{array}{c}10,9 \\
1,8 \quad 6\end{array}$ & $\begin{array}{ll} & 9,3 \\
1,3 & 6\end{array}$ \\
\hline $\begin{array}{l}\text { Xanthophyllum } \\
\text { papuamum }\end{array}$ & $\begin{array}{c}47,7 \\
6.6 \quad 22\end{array}$ & $\begin{array}{r}45,4 \\
3.79\end{array}$ & $\begin{array}{c}52,4 \\
5.2\end{array}$ & $\begin{array}{l}47,3 \\
8.8 \quad 8\end{array}$ & $1.7 \quad 21$ & $\begin{array}{l}15,1 \\
1.7\end{array}$ & $\begin{array}{l}12,7 \\
1.4\end{array}$ & $\begin{array}{c}13,2 \\
1.1\end{array}$ \\
\hline
\end{tabular}

${ }^{\mathrm{a}}$ Mean; ${ }^{\mathrm{b}}$ Standard deviation; ${ }^{\mathrm{c}}$ Number of specimens *not applicable

Table 5: Shear parallel to the grain and hardness per species based on position in the tree (based on height from ground).

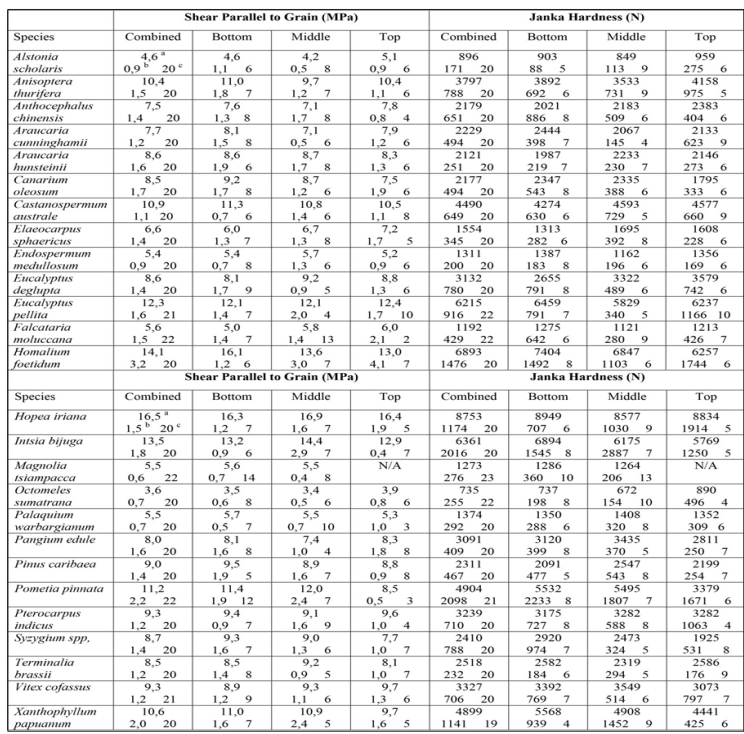

${ }^{\mathrm{a}}$ Mean; ${ }^{\mathrm{b}}$ Standard deviation; ${ }^{\mathrm{c}}$ Number of specimens. 
The position in the tree also had a limited effect on shear and hardness testing results (Table 5). The model only identified species as a very significant factor $(P$-value $<0,001)$ influencing shear strength and hardness. The Fisher pairwise comparisons analysis allowed identifying five species where hardness was significantly lower in top sections than bottom sections, i.e. Xanthophyllum papuanum, Homalium foetidum, Intsia bijuga, Syzygium spp., and Pometia pinnata. In the case of shear strength, four species could be identified as having significantly lower values in top sections versus bottom sections, i.e. Canarium oleosum, Homalium foetidum, Pometia pinnata, and Syzygium spp.. Like bending strength results, such a trend was usually noticeable in mid to high strength species.

\section{Comparison between old-growth forests and regrowth forests or plantations}

The mechanical properties of species obtained from plantations and regrowth forests were on average $24 \%$ lower than those from old-growth forests (Table 6, Bolza and Kloot 1976). However, it is not clear whether Bolza and Kloot (1976) used a primary ( $50 \times 50 \mathrm{~mm}$ specimens) or secondary ( $25 \times 25 \mathrm{~mm}$ or $20 \times 20 \mathrm{~mm}$ ) method for smaller size specimens. The authors also noted the extremely limited amount of test material to prepare specimens for many of the tested species, which suggested that smaller size specimens might have been considered here. Therefore, a length effect (Madsen 1990) where natural growth characteristics create cross sections with varying strengths along the length of a timber member might explain the lower mean values obtained in the present study. There was also no indication of tree age in the case of Bolza and Kloot (1976) which might have influenced the observed differences.

Octomeles sumatrana (-43\%), Magnolia tsiampacca (-40\%), and Syzygium spp. (-39\%) were the most affected species when comparing mechanical testing results with their counterparts from old-growth forests. On the other end, Anisoptera thurifera (+4\%), Elaeocarpus sphaericus (-10\%), and Araucaria hunsteinii (-11\%) were the least affected. The density variability observed between old-growth and regrowth or plantations timbers also support the differences observed between mechanical testing results. Octomeles sumatrana $(-22 \%)$, Magnolia tsiampacca (-27\%), and Syzygium spp. (-36\%) were again some the species most affected a density reduction where Anisoptera thurifera (+7\%), Araucaria hunsteinii ( $+5 \%)$, and Elaeocarpus sphaericus $(0 \%)$ showed an increase or no difference. Overall, the density of plantations and regrowth forests timbers was $11 \%$ lower compared with old-growth forests. However, density alone does not seem to explain all the variability observed and other elements such as ring width might need to be considered (Kliger et al. 1995). Barr et al. (2015) also observed a weak correlation between the density and the bending strength for Intsia bijuga.

Table 6: Physical and mechanical properties of Papua New Guinea species from old-growth forests (Bolza and Kloot 1976).

\begin{tabular}{|c|c|c|c|c|c|c|c|}
\hline & ADD & MOE & MOR & $\mathrm{Co}$ & ession & Shear & Hardness \\
\hline & $\begin{array}{c}\text { at } \\
12 \% \mathrm{MC}\end{array}$ & & & $\begin{array}{l}\text { Parallel to } \\
\text { grain }\end{array}$ & $\begin{array}{l}\text { Perpendicular } \\
\text { to grain }\end{array}$ & Parallel to grain & Janka \\
\hline Species & $\mathrm{kg} / \mathrm{m}^{3}$ & GPa & MPa & MPa & MPa & MPa & $\mathbf{N}$ \\
\hline Alstonia scholaris & 384 & 9,1 & 59,6 & 28,7 & $\mathrm{~N} / \mathrm{A}$ & 6,4 & 1736 \\
\hline Anisoptera thurifera & 641 & 13,3 & 79,3 & 44,3 & 8,4 & 11,9 & 4406 \\
\hline $\begin{array}{l}\text { Anthocephalus } \\
\text { chinensis }\end{array}$ & 465 & 9,8 & 74,5 & 41,3 & N/A & 11,3 & N/A \\
\hline Araucaria hunsteinii & 449 & 11,9 & 76,5 & 43,9 & 8,5 & 9,7 & 2392 \\
\hline Canarium oleosum & 561 & 11,7 & 89,6 & 49,0 & N/A & 12,8 & 3760 \\
\hline $\begin{array}{l}\text { Elaeocarpus } \\
\text { sphaericus }\end{array}$ & 384 & 7,9 & 56,6 & 32,5 & N/A & 8,7 & $\mathrm{~N} / \mathrm{A}$ \\
\hline $\begin{array}{l}\text { Endospermum } \\
\text { medullosum }\end{array}$ & 384 & 9,6 & 61,4 & 35,9 & 10,2 & 5,2 & 1713 \\
\hline Eucalyptus deglupta & 673 & 14,1 & 105,5 & 69,6 & 14,5 & 10,7 & 5340 \\
\hline Homalium foetidum & 897 & 19,2 & 151,7 & 84,1 & N/A & 24,2 & 9924 \\
\hline Hopea iriana & 977 & 24,2 & 168,9 & 94,5 & 28,3 & 18,4 & 10213 \\
\hline Intsia bijuga & 817 & 18,0 & 146,9 & 80,7 & 26,5 & 17,5 & 8566 \\
\hline Magnolia tsiampacca & 465 & 9,8 & 79,3 & 45,3 & N/A & 11,2 & $\mathrm{~N} / \mathrm{A}$ \\
\hline Octomeles sumatrana & 352 & 8,2 & 53,1 & 36,3 & 5,8 & 5,8 & 1580 \\
\hline Pometia pimnata & 689 & 14,3 & 106,2 & 59,8 & 15,6 & 14,4 & 6542 \\
\hline Pterocarpus indicus & 609 & 12,2 & 95,1 & 58,3 & 16,6 & 9,9 & 4,695 \\
\hline Syzygium spp. & 769 & 16,3 & 110,3 & 67,7 & N/A & 13,7 & $\mathrm{~N} / \mathrm{A}$ \\
\hline Terminalia brassii & 465 & 9,9 & 68,1 & 37,2 & 10,0 & 8,2 & 2759 \\
\hline Vitex cofassus & 705 & 13,6 & 113,1 & 63,7 & 17,9 & 16,3 & 5585 \\
\hline $\begin{array}{l}\text { Xanthophyllum } \\
\text { papuamum }\end{array}$ & 785 & 18,5 & 130,3 & 78,6 & N/A & 15,0 & 7432 \\
\hline
\end{tabular}

ADD: Air dry density at $12 \%$ moisture content; MOE: Modulus of elasticity or stiffness; MOR: Modulus of rupture or bending strength. Araucaria cunninghamii, Falcataria moluccana, Castanospermum austral, Eucalyptus pellita, Palaquium warbargianum, Pangium edule, Pinus caribaea are not included in the list of studied species by Bolza and Kloot (1976). 
Anisoptera thurifera was the only species from regrowth forests where the mechanical testing results were usually higher $(+4 \%)$ across all selected mechanical properties than those from old-growth forests (Bolza and Kloot 1976). In the case of plantations species, Magnolia tsiampacca (-40\%) and Eucalyptus deglupta (-35\%) were the species showing the most important average decrease in terms of mechanical properties when compared with old-growth forests. Araucaria hunsteinii (-11\%) and Terminalia brassii (-17\%) were the two plantations species least affected when compared with their old-growth counterparts. Compression perpendicular to the grain $(-31 \%$ on average across all species), hardness $(-30 \%)$, and compression parallel to the grain $(-29 \%)$ were the properties showing the highest drops when comparing with old-growth forests timbers. The bending stiffness $(-23 \%)$ was the mechanical property least affected when compared with results from old-growth forests followed by bending strength and shear (-24\% for both).

A study by Edwin and Ozarska (2015) support the observed decrease in mechanical properties of PNG timbers from regrowth forests and plantations when compared with old-growth forests. The physical and bending properties results of 6 hardwoods species from secondary forests in PNG are also in accordance with those obtained in the present study. A study by Haslett et al. (1991) on Anthocephalus chinensis from West Samoa also provided physical and bending properties fitting those of the present study with stiffness and bending strength of $6,8 \mathrm{GPa}$ and $58 \mathrm{MPa}$, respectively, for an ADD of $340 \mathrm{~kg} / \mathrm{m}^{3}$. A lower compression parallel to the grain (25 MPa) might be related to a site factor for growth traits as reported by Leksono et al. 2008 and Hung et al. (2015) for Eucalyptus pellita. Multiple studies on Eucalyptus pellita also demonstrated the site impact on timber mechanical properties (Bootle 2005, Kelin et al. 2006, Hung et al. 2015). Interlocked grain as an adaptive trait for tropical tree species in the rainforest has also been suggested by Cabrolier et al. (2009) after the authors noticed strong variations between and within trees. Where special attention was taken in the selection of specimens for the present study, the presence of interlocked grain could explain some of the differences observed between old-growth and secondary growth timbers. Barr et al. (2015) studied the impact of interlocked grain on bending properties of Intsia bijuga. The authors noted that the grain can vary significantly from straight to interlocked and influence significantly the bending strength. Interestingly, stiffnesses and densities found in the present study are similar to those found by Barr et al. 2015 i.e. 758 and $812 \mathrm{~kg} / \mathrm{m}^{3}$ and 14,2 and 15 $\mathrm{GPa}$, respectively. However, MOR values differed significantly (116,6 versus $152,5 \mathrm{MPa})$ potentially because of the presence of interlocked grain.

\section{CONCLUSIONS}

Six mechanical properties, namely bending strength, stiffness, compression parallel and perpendicular to the grain, shear parallel to the grain, and hardness, have been evaluated for 26 PNG species using 2641 small clear specimens from 130 trees.

The impact of the position in the tree on the selected mechanical properties has also been assessed. Stiffness and bending strength tend to decrease or remain unchanged along the stem across all studied species. Where shear and hardness testing results showed a similar trend to a lesser extent, the position in the tree had a much more limited impact on the compression strength properties. Further experiments where sampling would consider the radial position within the tree might accentuate observed trends. Therefore, segregating logs based on the position in the tree could be of interest where desired timber mechanical properties and costs associated with segregating is justifying optimum mechanical properties for the intended end use.

The mechanical properties of species obtained from plantations and regrowth forests were lower than those found in the literature from old-growth forests. Different factors including the size of specimens tested, the amount and provenance of tested material, and some adaptive traits for tropical tree species might explain some differences. However, comparisons of mechanical testing results with other recent studies tend to confirm a reduction of physical and mechanical properties when comparing with timbers from old-growth forests. 


\section{ACKNOWLEDGMENTS}

The authors acknowledge the financial support of the Australian Government for funding through the Australian Centre for International Agricultural Research (ACIAR) program, Project No. FST/2012/092. Gratitude is extended to the Timber and Forestry Training College and PNG Forest Research Institute for harvest and supply of material used in the study. Special thanks to Frank Asok, Markson Naki, and Grace So'on for their assistance with experimental and coordination activities.

\section{REFERENCES}

ASTM International. 2009. Standard test methods for small clear specimens of timber. ASTM D143. 2009. ASTM International: United States.

ASTM International. 2010. Standard Practice for Sampling Forest Trees for Determination of Clear Wood Properties. ASTM D5536. 2010. ASTM International: United States.

Barr, J.; Tippner, J.; Rademacher, P. 2015. Prediction of mechanical properties - Modulus of rupture and modulus of elasticity - of five tropical species by non-destructive methods. Maderas-Cienc Tecnol 17(2):239252. http://dx.doi.org/10.4067/S0718-221X2015005000023.

Barrett, J.D.; Kellogg, R.M. 1991. Bending strength and stiffness of second-growth Douglas-fir dimension lumber. Forest Prod J 41 (10): 35-43.

Bendtsen, B.A. 1978. Properties of wood from improved and intensively managed trees. Forest Prod $J$ 28(10):61-72.

Bolza, E.; Kloot, N.H. 1976. The mechanical properties of 81 New Guinea timbers. Technical Paper No. 11, Division of Building Research, CSIRO: Melbourne, Australia.

Bootle, K.R. 2005. Wood in Australia. $2^{\text {nd }}$ Edition. McGraw-Hill: Australia. ISBN: 0071014012

Cabrolier, P.; Beauchene, J.; Thibaut, B. 2009. Is interlocked grain an adaptive trait for tropical tree species in rainforest?. $6^{\text {th }}$ Plant Biomechanics Conference. Cayenne, French Guyana, 16-21-nov, 2009. pp.279284.

Edwin, P.; Ozarska, B. 2015. Bending properties of hardwood timbers from secondary forest in Papua New Guinea. Journal of Tropical Forest Science 27(4): 456-461.

FAO. 2015. Global Forest Resources Assessment 2015. Food and Agriculture Organization of the United Nations: Rome, Italy. 253 p. ISBN 978-92-5-108826-5.

Harvald, C. 1988. Nåletraearternes tekniske egenskaper (Mechanical properties of softwoods, in Danish). Royal Veterinary and Agricultural Univ., Dept. of Forestry, Copenhagen, Denmark

Haslett, A.N.; Young, G.D.; Britton, R.A.J. 1991. Plantation grown tropical timbers. 2. Properties, processing and uses. Journal of Tropical Forest Science 3(3):229-237.

Hojbo, O.A. 1991. The quality of wood of Norway spruce (Picea abies) planted with different spacing. Ph.D. Thesis, Agricultural University of Norway, Norway. ISBN: 82-575-0141-7

Hung, T.D.; Brawner, J.T.; Meder, R.; Lee, D.J.; Southerton, S.; Thinh, H.H.; Dieters, M.J. 2015. Estimates of genetic parameters for growth and wood properties in Eucalyptus pellita F. Muell. to support tree breeding in Vietnam. Annals of Forest Science 72:205-217. https://doi.org/10.1007/s13595-014-0426-9.

Kelin, Y.; Xiaomei, J.; Jianxiong, L. 2006. Guide on utilization of eucalyptus and acacia plantations in China for solid wood products. Research Institute of Wood Industry. Chinese Academy of Forestry. Technical Report. Science Press: China. ISBN :7030194004. 195 p.

Kotlarewski, N.J.; Belleville, B.; Gusamo, B.K.; Ozarska, B. 2016. Mechanical properties of Papua 
New Guinea balsa wood. European Journal of Wood and Wood Products 74:83-89. https://doi.org/10.1007/ s00107-015-0983-0.

Kliger, I.R.; Perstorper, M.; Johansson, G.; Pellicane P.J. 1995. Quality of timber products from Norway spruce - Part 3. Influence of spatial position and growth characteristics on bending stiffness and strength. Wood Sci Technol 29: 397-410. https://doi.org/10.1007/BF00194198.

Leksono, B.; Kurinobu, S.; Ide, Y. 2008. Realized genetic gains observed in second generation seedling seed orchards of Eucalyptus pellita in Indonesia. J For Res 13:110-116. https://doi.org/10.1007/s10310-0080061-0.

Madsen, B. 1990. Length effect in 38 mm spruce-pine-fir dimension lumber. Can J Civ Eng 17: 226-242.

Machado, J.; Cruz, H. 2005. Within stem variation of maritime pine timber mechanical properties. Holz Roh Werkst 63(2): 154-159. https://doi.org/10.1007/s00107-004-0560-4.

Pearson, R.G.; Gilmore, R.C. 1980. Effects of fast growth rate on the mechanical properties of Loblolly pine. Forest Prod J 30(5): 60-66.

PNGFA. 2007. Overview of PNG's Forests. Papua New Guinea Forest Authority. <http://www.forestry. gov.pg> (Accessed 3 February 2017).

Shivnaraine, C.S. 1989. Within stem variation in bending strength and stiffness of lumber from plantation grown white spruce. M.Sc. Thesis, University of New Brunswick, Fredericton, Canada. 